\title{
Bistability driven by colored noise: Theory and experiment
}

\author{
Peter Hanggi \\ Department of Physics, Polytechnic Institute of New York, Brooklyn, New York 11201
}

Thomas J. Mroczkowski and Frank Moss

Department of Physics, University of Missouri-St. Louis, St Louis, Missouri 63121

P. V. E. McClintock

Department of Physics, University of Lancaster, Lancaster, LA1 4YB, United Kingdom

(Received 23 January 1985)

\begin{abstract}
A nonequilibrium, bistable flow driven by exponentially correlated Gaussian noise is considered. An approximate, nonlinear Fokker-Planck-type equation, modeling effectively the long-time dynamics of the bistable, non-Markovian flow is constructed, and the mean sojourn time is evaluated in the limit of weak noise. Keeping the noise strength constant, the mean sojourn time is predicted to undergo an exponential increase with increasing noise-correlation time. Representing the bistable, colored noise dynamics with an electronic circuit, the mean of the sojourn time and the sojourn-time distribution have been measured experimentally. The experiments confirm the exponential increase for the mean sojourn time and close quantitative agreement with this newly proposed theoretical approach is found. In contrast, previous approximation schemes which expand around the Markovian theory (zero-noise correlation time) would predict for this case an exponentially decreasing mean sojourn time upon increasing the noise-correlation time, in marked disagreement with the present measurements.
\end{abstract}

Over the last years, there has been a steadily growing interest in nonlinear systems in which the nonlinear coupling induces instabilities and bistability within certain ranges of the control parameter regime. ${ }^{1}$ Moreover, one now realizes that the role of fluctuations is far less trivial in systems far from equilibrium than in systems in thermodynamic equilibrium. ${ }^{2-7}$ One of the more puzzling enigmas that has emerged recently is the observation that the weak noise dynamics of Fokker-Planck systems in state-space dimension $d \geqslant 2$ is generally beset with trouble which can be related to erratic, chaotic behavior. ${ }^{8}$ One such model system exhibiting bistability is the two-dimensional Fokker-Planck dynamics $^{9}$

$$
\begin{aligned}
\dot{p}_{t}(x, y)= & +\frac{\partial}{\partial x}\left[V^{\prime}(x) p_{t}(x, y)\right]-y \frac{\partial}{\partial x} p_{t}(x, y) \\
& +\frac{1}{\tau} \frac{\partial}{\partial y}\left[y p_{t}(x, y)\right]+\frac{D}{\tau^{2}} \frac{\partial^{2}}{\partial y^{2}} p_{t}(x, y),
\end{aligned}
$$

where $V(x)$ denotes the symmetric bistable potential field

$$
V(x)=-\frac{a}{2} x^{2}+\frac{b}{4} x^{4}, a>0, b>0
$$

and where $V^{\prime}(x)$ is its derivative. The dynamics in (1) does not obey detailed balance, ${ }^{3,4}$ which in practice renders explicit analytical studies impossible. Following Ref. 9, we impose correlation-free initial conditions, i.e., the initial probability $p_{0}(x, y)$ factorizes in the variables $x$ and $y$, and use for $y(t=0)$ a stationary Gaussian with mean value zero and variance $\left\langle y^{2}\right\rangle=D / \tau$. Then, the dynamics in (1) can be mapped onto the one-dimensional, but non-Markovian, Langevin equation driven by additive, exponentially correlated Gaussian noise

$$
\begin{aligned}
& \dot{x}=a x-b x^{3}+\xi(t), \\
& \langle\xi(t) \xi(s)\rangle=\frac{D}{\tau} \exp (-|t-s| / \tau) .
\end{aligned}
$$

The rate of change of the single-event probability $p_{t}(x)$ of the bistable, non-Markovian flow (3) then obeys the formally exact equation ${ }^{9,10}$

$$
\dot{p}_{t}(x)=-\frac{\partial}{\partial x}\left(a x-b x^{3}\right) p_{t}+\frac{D}{\tau} \frac{\partial^{2}}{\partial x^{2}} \int_{0}^{t} d s \exp [-(t-s) / \tau]\left\langle\delta(x(t)-x) \frac{\delta x(t)}{\delta \xi(s)}\right\rangle,
$$

where the functional derivative $[\delta x(t) / \delta \xi(s)]$ obeys the integral equation

$$
\frac{\delta x(t)}{\delta \xi(s)}=\theta(t-s)\left(1+\int_{s}^{t} d \tau\left[a-3 b x^{2}(\tau)\right] \frac{\delta x(\tau)}{\delta \xi(s)}\right) .
$$

At this point, it becomes clear that all of our original difficulties are only being transferred to an evaluation of the nonlinear functional in (5). Upon a repeated use of (5) one can rewrite the average in the second term in (4) in the form

$$
\left\langle\delta(x(t)-x)\left[1+\int_{s}^{t} d \tau_{1}\left(a-3 b x^{2}\left(\tau_{1}\right)\right)\left(1+\int_{s}^{\tau_{1}} d \tau_{2}\left(a-3 b x^{2}\left(\tau_{2}\right)\right)(1+\cdots)\right) \cdots\right]\right\rangle .
$$


If we now neglect transients (i.e., we let $t \rightarrow \infty$ ), and make use of a decoupling ansatz, thereby neglecting induced correlations $^{10}$ among the successive functionals in (6) we end up with an effective "nonlinear" (approximate) Fokker-Planck dynamics

$$
\begin{aligned}
\dot{p}_{t}(x)= & -\frac{\partial}{\partial x}\left(a x-b x^{3}\right) p_{t}(x) \\
& +\frac{D}{1+\tau\left(3 b\left\langle x^{2}\right\rangle-a\right)} \frac{\partial^{2}}{\partial x^{2}} p_{t}(x),
\end{aligned}
$$

where the quantity $\left\langle x^{2}\right\rangle$ depends itself on the (unknown) solution $p_{t}(x)$. Clearly, this type of "nonlinear" approximation will work best for a small correlation time $\tau$ and converges for $\tau=0$ to the well-known Smoluchowski equation $^{3,4,6,7}$ (the Gaussian white noise limit). Because the stationary second moment ${ }^{11}\left\langle x^{2}\right\rangle_{\tau=0}$ changes for a symmetric double well potential only little as $D$ is varied (e.g., with $a=b=1$, one obtains $\quad D=0: \quad\left\langle x^{2}\right\rangle=1, \quad D=0.1$ : $\left.\left\langle x^{2}\right\rangle=0.87, D=1:\left\langle x^{2}\right\rangle=1.01, D=100:\left\langle x^{2}\right\rangle=1.34\right)$ we can identify for small $\tau$ the parameter $\left\langle x^{2}\right\rangle$ with the white noise result. Note also that $\left\langle x^{2}\right\rangle \simeq a / b$; therefore, the effective diffusion coefficient in Eq. (7) is always positive.

A quantity which sensitively probes the form of the wings of the stationary probability is the mean sojourn time $\langle T\rangle$ in the stable state. For small-noise intensity $D$, the trajectory $x(t)$ [see Fig. 1(b)] stays for a long time around one of the stable minima before eventually crossing over into the neighboring well. Owing to such a clear-cut time separation, those successive jumps back and forth between the two wells can be looked upon as independent random events; i.e., the sojourn times $T$ are exponentially distributed as ${ }^{12}$

$$
p(T)=\frac{1}{\langle T\rangle} \exp (-T /\langle T\rangle) .
$$

The mean sojourn time $\langle T\rangle$ is exponentially large and its

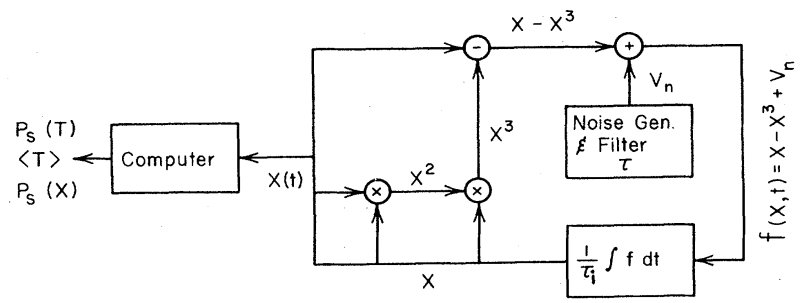

(a)

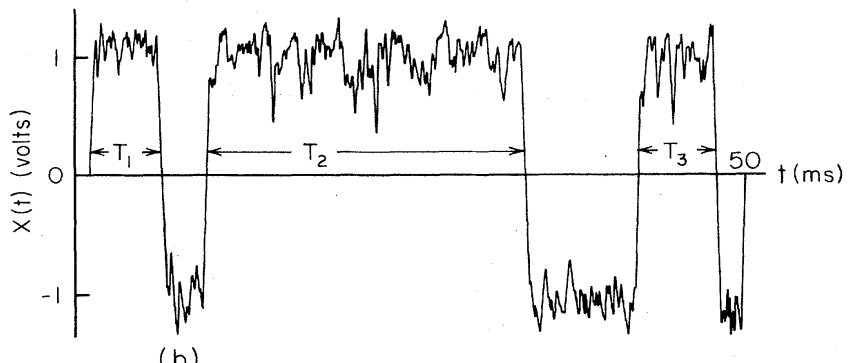

(b)

FIG. 1 (a) A schematic diagram of the circuit. All summation and multiplication operations are performed with standard, commercially available analog components. The computer functions only as a measuring instrument. (b) An example time function, measured at the integrator output. leading behavior depends only on the leading behavior of the ratio $R$ of the stationary probability $\bar{p}$, taken at the stable states $x_{1 / 2}=\mp(a / b)^{1 / 2}$ and the unstable state $x=0$, i.e.,

$$
\langle T\rangle \propto R=\bar{p}\left(x=-(a / b)^{1 / 2}\right) / \bar{p}(x=0) .
$$

Because (7) models effectively the long-time dynamics of the non-Markovian process $x(t)$, one obtains for the leading behavior of the mean sojourn time $e^{6,9}$ of (7) (by use of the method of steepest descent)

$$
\langle T\rangle=\frac{\pi}{a \sqrt{2}} \exp \frac{\Delta \phi(\tau)}{D},
$$

where

$$
\Delta \phi=\left(a^{2} / 4 b\right)\left[1+\tau\left(3 b\left\langle x^{2}\right\rangle-a\right)\right] .
$$

Thus, the "nonlinear," somewhat crude, approximation scheme in (7) predicts an exponential increase for $\langle T\rangle$ with increasing noise-correlation time $\tau .{ }^{13}$ In contrast, using the usual approximation scheme, 6,14 which expands the functional in (5) around the Markovian theory, one predicts that $\Delta \phi(\tau)$ actually decreases in proportion to $\tau^{2}$ with increasing noise-correlation time (no uniform convergence to the white noise limit). ${ }^{9}$

We have found Eqs. (9) and (10) to be in quite good agreement with measurements of $\langle T\rangle$ obtained using an analog electronic circuit similar to ones we have used previously. ${ }^{15}$ The circuit, which is shown in Fig. 1(a), approximately solves

$$
x(t)=\left(1 / \tau_{i}\right) \int\left(a^{\prime} x-b^{\prime} x^{3}+V_{n}\right) d t .
$$

The integrand is Eq. (3a) scaled by $\tau_{i}$, the integrator time constant, with $a^{\prime} / \tau_{i}=a, b^{\prime} / \tau_{i}=b$, and $V_{n} / \tau_{i}=\xi(t)$. Throughout this experiment $\tau_{i}=100 \mu \mathrm{s}$. The scaled, mean-square noise intensity is then given by

$$
\left\langle V_{n}^{2}\right\rangle / \tau_{i}^{2}=D^{\prime} / \tau,
$$

where $\left\langle V_{n}^{2}\right\rangle$ was measured at the output of the noise filter. The circuit was designed with an overall scale factor of unity so that measurements of $x(t)$ at the integrator output in volts correspond numerically to solutions of Eq. (11).

An example output (realization) is shown in Fig. 1(b), where random switching between potential wells whose minima are near $\pm 1 \mathrm{~V}$ is evident. The realizations $x(t)$ are digitized by the computer, and the sojourn times $T_{i}$ are measured between threshold crossings near zero as shown. The computer then assembles the density $p(T)$ which approaches the stationary density $\bar{p}(T)$ for a sufficiently large number of samples. Our measurements of $\bar{p}(T)$ are quite accurately represented by Eq. (8). The mean sojourn time is then obtained from

$$
\langle T\rangle=\sum_{i=1}^{N} T_{i} \bar{p}\left(T_{i}\right),
$$

where $N$ was typically 1000 . A sequence of measurements of $\langle T\rangle$ was made by varying $\tau$, while holding $D^{\prime}$ constant. These results are shown in Fig. 2, where it is obvious that $\langle T\rangle$ rapidly increases with $\tau$ for all observations. For all data, $\tau<<\langle T\rangle$, so that successive jumps occur independently. [This condition is necessary in order to ensure that $\bar{p}(T)$ is accurately exponential.] The scatter for each set of data is representative of the statistical errors. 


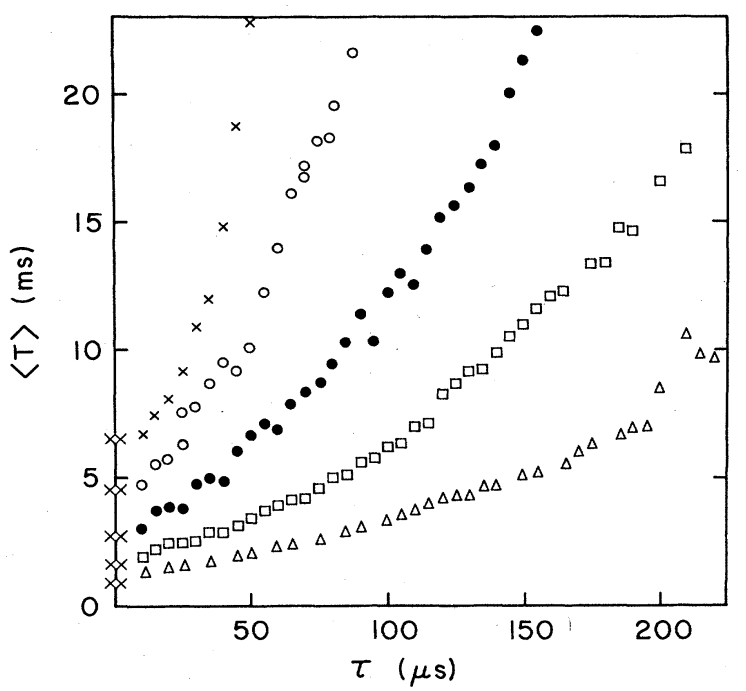

FIG. 2. Measured values of $\langle T\rangle$ vs $\tau$ for several values of the unscaled, dimensionless noise intensity $D$ : crosses: 0.073 , open circles: 0.083 , closed circles: 0.114 , squares: 0.153 , and triangles: 0.212 . The scaled noise intensity is obtained from Eq. (12). The theoretical white noise limits, predicted by Eqs. (9) and (10) with no adjustable parameters, are indicated by the lozenges.

In order to compare these results with the theory, we have obtained $\Delta \phi$ for each datum point using the scaled version of Eq. (9b). The data were then replotted on Fig. 3, where the solid lines are fits to the scaled version of Eq. (10):

$$
\Delta \phi=\left(1 / 4 \tau_{i}\right)\left[1+\left(\tau / \tau_{i}\right)\left(3\left\langle x^{2}\right\rangle-1\right)\right],
$$

for $a^{\prime}=b^{\prime}=1$, using $\left\langle x^{2}\right\rangle$ as the only adjustable parameter. Fits were made to the triangles and closed circles as shown by the solid lines in Fig. 3. As a test of the theory we have calculated $\left\langle x^{2}\right\rangle$ from the white noise, scaled density function

$$
\bar{p}^{\prime}(x)=N \exp \left(\tau_{i} / \tau\left\langle V_{n}^{2}\right\rangle\right)\left(x^{2} / 2-x^{4} / 4\right)
$$

by numerical integration, with the result that $\left\langle x^{2}\right\rangle_{\text {calc }}=0.83 V^{2}$ and $0.86 V^{2}$ for the triangles and closed circles, respectively. The experimentally obtained values are $0.84 \mathrm{~V}^{2}$ and $0.60 \mathrm{~V}^{2}$, respectively (see Fig. 3). Note that the closed circles (Fig. 3) correspond to a lower noise intensity, i.e., stronger exponential suppression of probability of the unstable state. This explains the expected larger difference

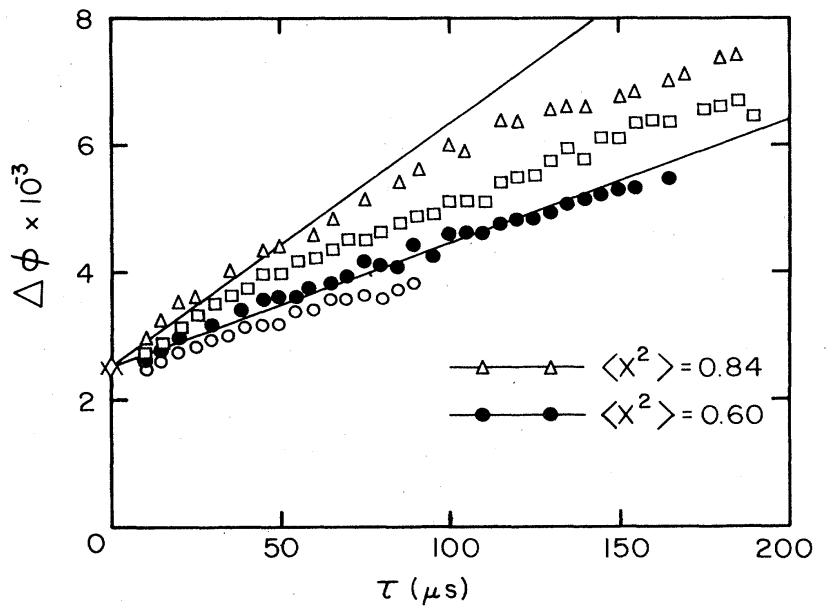

FIG. 3. The data are replotted to show $\Delta \phi$ vs noise-correlation time $\tau$. The solid lines are Eq. (14) for the values of $\left\langle x^{2}\right\rangle$ shown. The lozenge indicates the white noise limit of Eq. (14), with no adjustable parameter.

between approximate, theoretical value and the experimental value. Keeping in mind that the approximations made leading to (7) are rather crude, the agreement found is more than satisfactory. Moreover, the close agreement between the measured density $\bar{p}(T)$ and (8) indicates that the measured escape is controlled by the Arrhenius factor given by (9a) and (10).

In this work we have shown that a finite noise-correlation time $\tau$ can complicate matters considerably, but yet can lead to important effects. With $\tau$ finite the (manifest) detailed balance of a one-dimensional Fokker-Planck equation is broken. This work then demonstrates that nonlinear approximation schemes of the type used in (7), which partially incorporate the complexities of the non-Markovian process $x(t)$, are essential in order to model accurately the colorednoise dynamics. In particular, this is true in bistable flows ${ }^{1-4}$ where the exponential suppression of probability of the locally unstable state depends sensitively on the details of the noise properties. Thus, these systems are ideal to test different theoretical approximation schemes for colored noise. ${ }^{10,14,16}$

The importance of colored noise has recently also been recognized in nonlinear optical systems which undergo a Hopf-bifurcation-type instability. ${ }^{17}$ The study of colored noise in optical bistable systems too, yields several such key results. $^{18}$
${ }^{1}$ For an item which reviews the recent advances and presents the state of the art in this field, see Fluctuations and Sensitivity in Nonequilibtrium Systems, edited by W. Horsthemke and D. K. Kondepundi, Springer Proceedings in Physics, Vol. I (Springer-Verlag, Berlin, 1984).

${ }^{2}$ G. Nicolis, Rep. Prog. Phys. 42, 225 (1979).

${ }^{3}$ P. Hanggi and H. Thomas, Phys. Rep. 88, 207 (1982).

${ }^{4} \mathrm{H}$. Risken, The Fokker-Planck Equation, Springer Series in Synergetics, Vol. 18 (Springer-Verlag, Berlin, 1984).

${ }^{5} \mathrm{~W}$. Horsthemke and R. Lefever, Noise Induced Transitions, Springer Series in Synergetics, Vol. 15 (Springer-Verlag, Berlin, 1984).
${ }^{6}$ R. L. Stratonovich, Topics in the Theory of Random Noise (Gordon and Breach, New York, 1963, 1966), Vols. I and II.

${ }^{7}$ R. Landauer, Phys. Today 31, 23 (1978); Helv. Phys. Acta 56, 847 (1983).

${ }^{8}$ R. Graham and T. Tel, J. Stat. Phys. 35, 729 (1984); Phys. Rev. Lett. 52, 9 (1984).

${ }^{9}$ P. Hanggi, F. Marchesoni, and P. Grigolini, Z. Phys. B 56, 333 (1984).

10P. Hanggi, Z. Phys. B 31, 407 (1978); in Stochastic Processes Applied to Physics, edited by L. Pesquera and M. Rodriquez (World Scientific, Singapore, 1985). 


\section{RAPID COMMUNICATIONS}

${ }^{11}$ For a symmetric double well, the stationary second moment $\left\langle x^{2}\right\rangle$ can be expressed in terms of parabolic cylinder functions $D_{v}$ [Handbook of Mathematical Functions, edited by M. Abramowitz and I. A. Stegun (Dover, New York, 1968), p. 687], as

$$
\left\langle x^{2}\right\rangle_{\tau=0}=\frac{1}{2}\left(\frac{2 D}{b}\right)^{1 / 2}\left(\left.\frac{D_{-3 / 2}}{D_{-1 / 2}}\right|_{x=-a /(2 b D)^{1 / 2}}\right) .
$$

${ }^{12}$ R. Roy, R. Short, J. Durnin, and L. Mandel, Phys. Rev. Lett. 45, 1486 (1980).

${ }^{13}$ This predicted behavior is in qualitative agreement with previous results for colored noise bistable flows driven by a symmetric two-state process [P. Hanggi and P. Riseborough, Phys. Rev. A 27, 3379 (1983); C. Van den Broeck and P. Hanggi, ibid. 30, 2730 (1984)].

${ }^{14}$ J. M. Sancho, M. San Miguel, S. L. Katz, and J. D. Gunton, Phys. Rev. A 26, 1589 (1982); M. Lax, Rev. Mod. Phys. 38, 541 (1966); N. G. Van Kampen, Phys. Rep. 24C, 171 (1976), pp. $210 \mathrm{ff}$.

15J. Smythe, F. Moss, and P. V. E. McClintock, Phys. Rev. Lett. 51, 1062 (1983); J. D. Robinson, F. Moss, and P. V. E. McClintock, J. Phys. A 18, L89 (1985).

${ }^{16}$ R. F. Fox, Phys. Lett. 94A, 281 (1983).

${ }^{17}$ R. Short, L. Mandel, and R. Roy, Phys. Rev. Lett. 49, 647 (1982); P. Lett, R. Short, and L. Mandel, ibid. 52, 341 (1984); R. F. Fox, G. E. James, and R. Roy, Phys. Rev. A 30, 2482 (1984).

${ }^{18} \mathrm{P}$. Hanggi and F. Moss, in Proceedings of the International Conference on Instabilities in Lasers and Nonlinear Optical Systems, Rochester, 1985 (unpublished). 